\title{
Heteroatom Induced Electronic Structure Modulation of Vertically Oriented Oxygen Vacancy-rich NiFe Layered Double Oxide Nanoflakes to Boost Bifunctional Catalytic Activity in Li- $\mathrm{O}_{2}$ Battery
}

Jiabao Li, Chaozhu Shu*, Zhiqun Ran, Minglu Li, Ruixin Zheng, Jianping Long *

College of Materials and Chemistry \& ChemicalEngineering, Chengdu University of Technology, 1\#, Dongsanlu, Erxianqiao, Chengdu 610059, Sichuan, P. R. China

*Email: czshu@imr.ac.cn;

*Email: longjianping@cdut.cn 


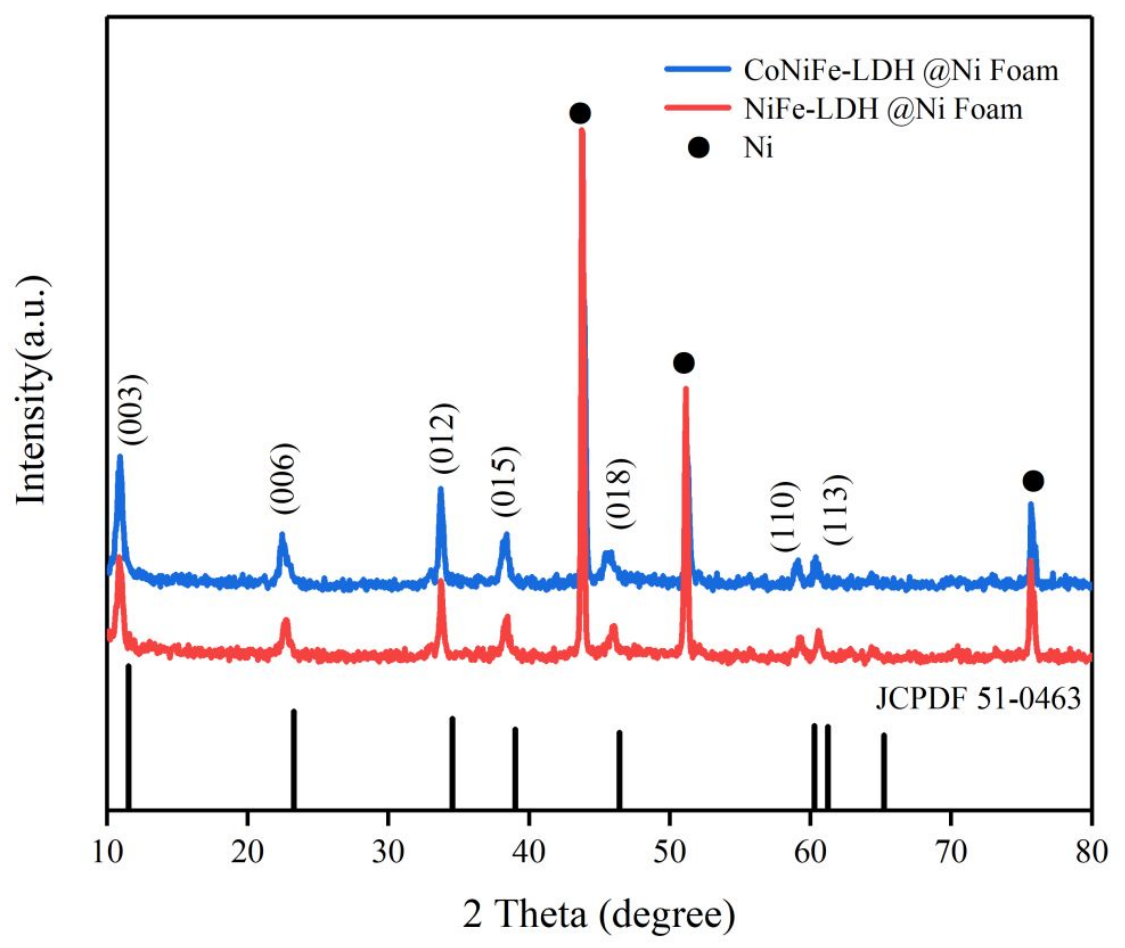

Figure S1. XRD pattern of CoNiFe LDH 

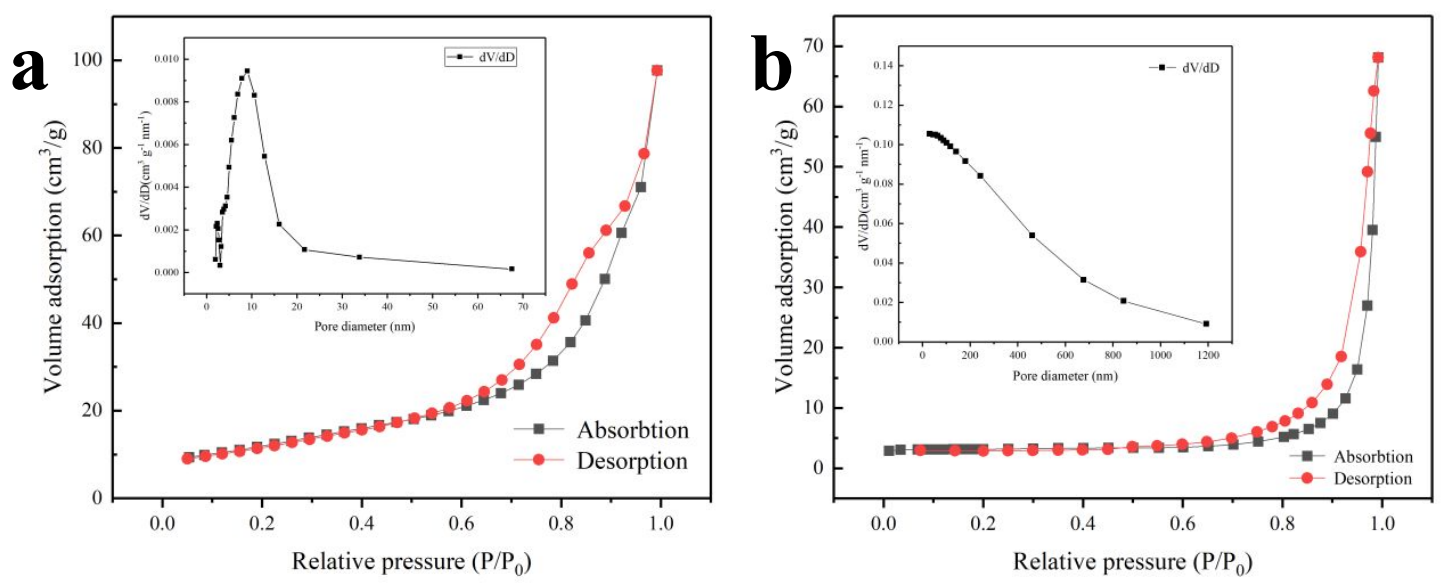

Figure S2. $\mathrm{N}_{2}$ sorption isotherms and the pore size distribution curve (inset) of

a) CoNiFe LDO and b) Co-NiFe LDH 

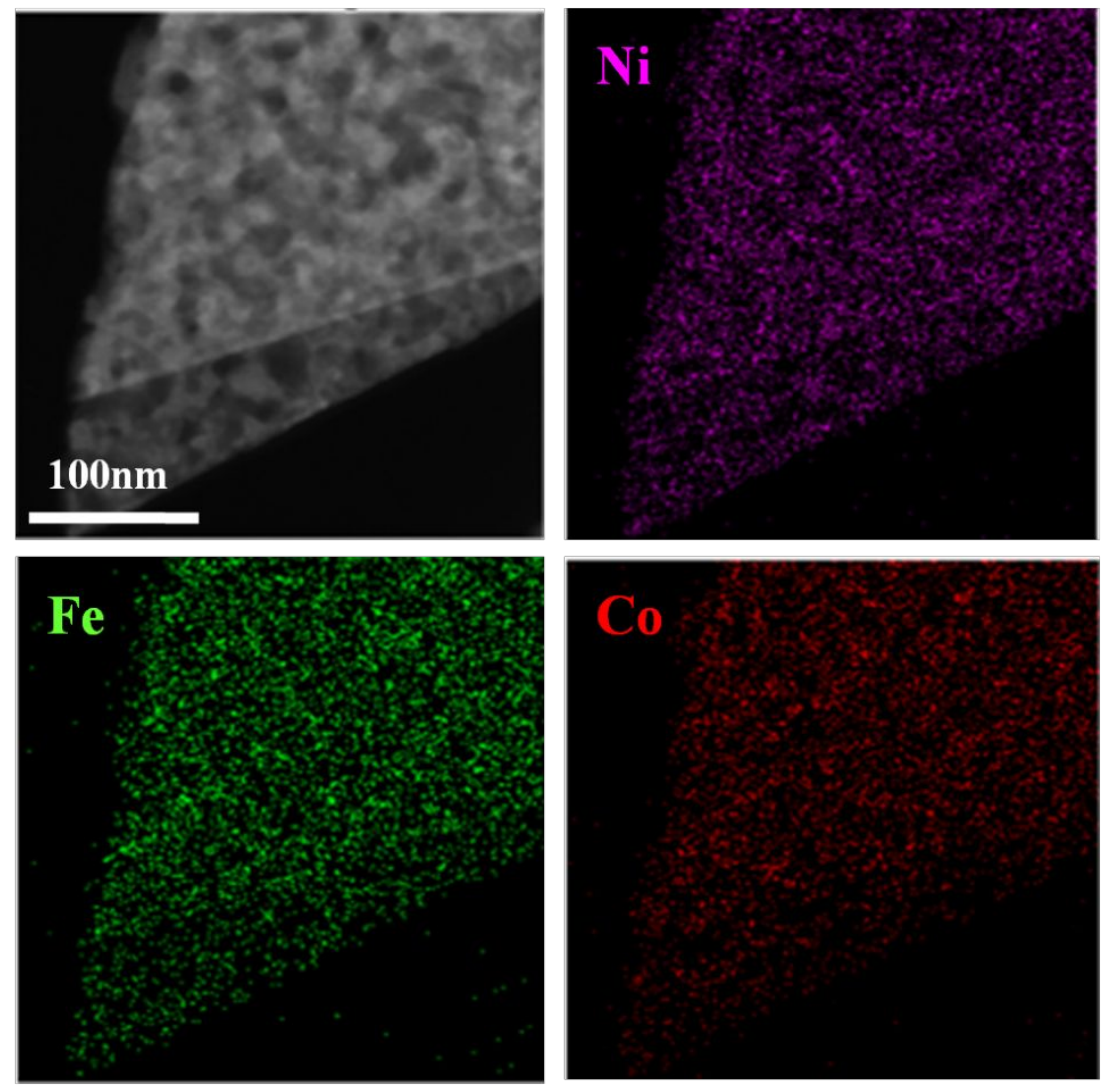

Figure S3. Element mapping of CoNiFe LDO
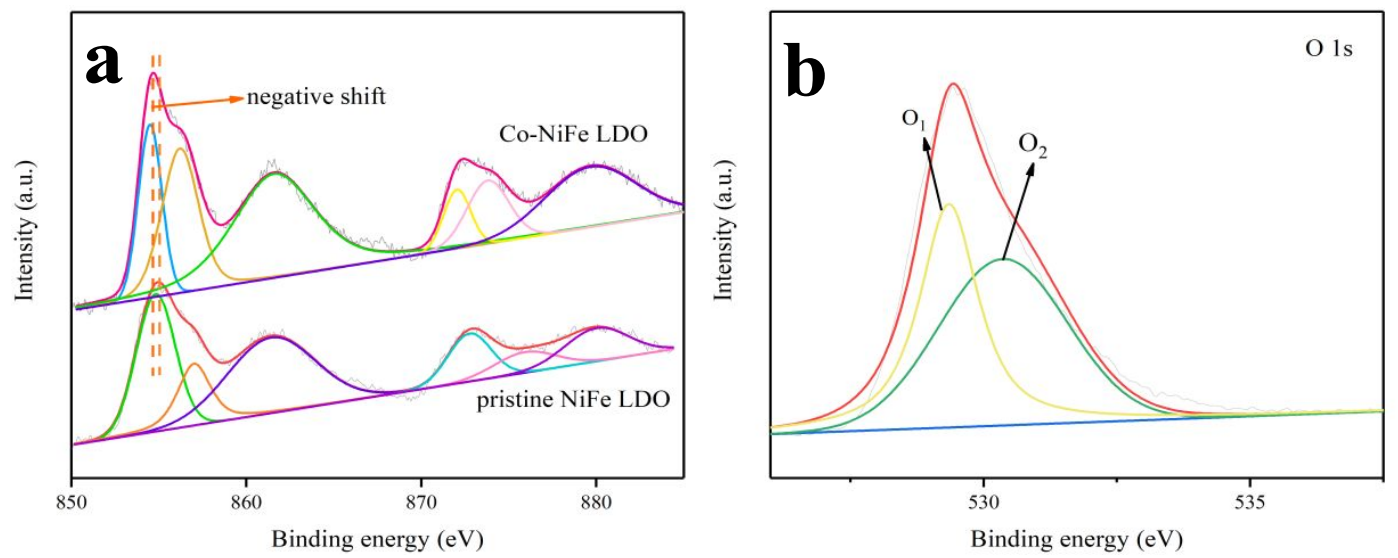

Figure S4. a) Ni $2 p$ spectrum pattern of NiFe LDO and CoNiFe LDO; b) O1s specrta of NiFe LDO@Ni foam 


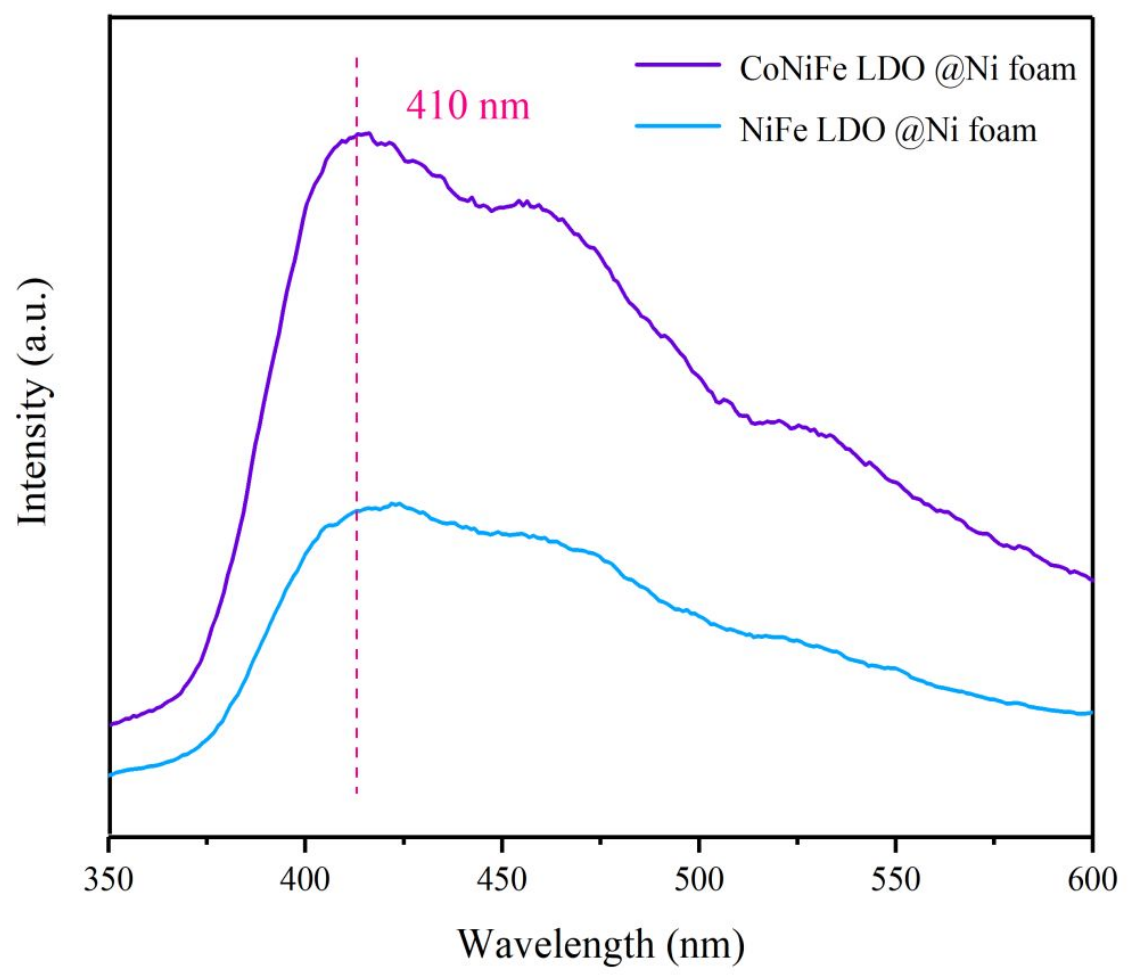

Figure S5. PL spectra for CoNiFe LDO@Ni foam and NiFe LDO@Ni foam

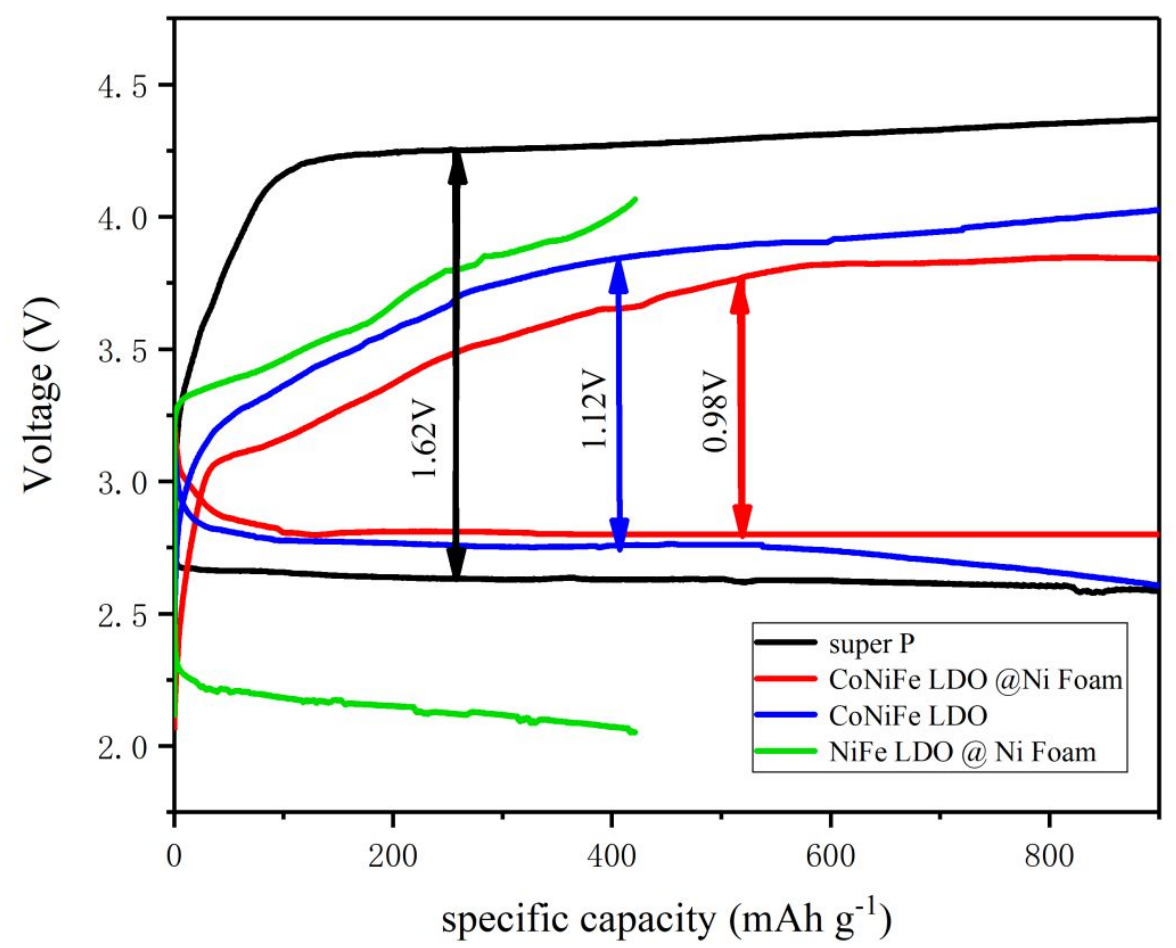

Figure S6. First charge discharge profile of super $p$, CoNiFe LDO, CoNiFe LDO@Ni foam, NiFe LDO@Ni foam 


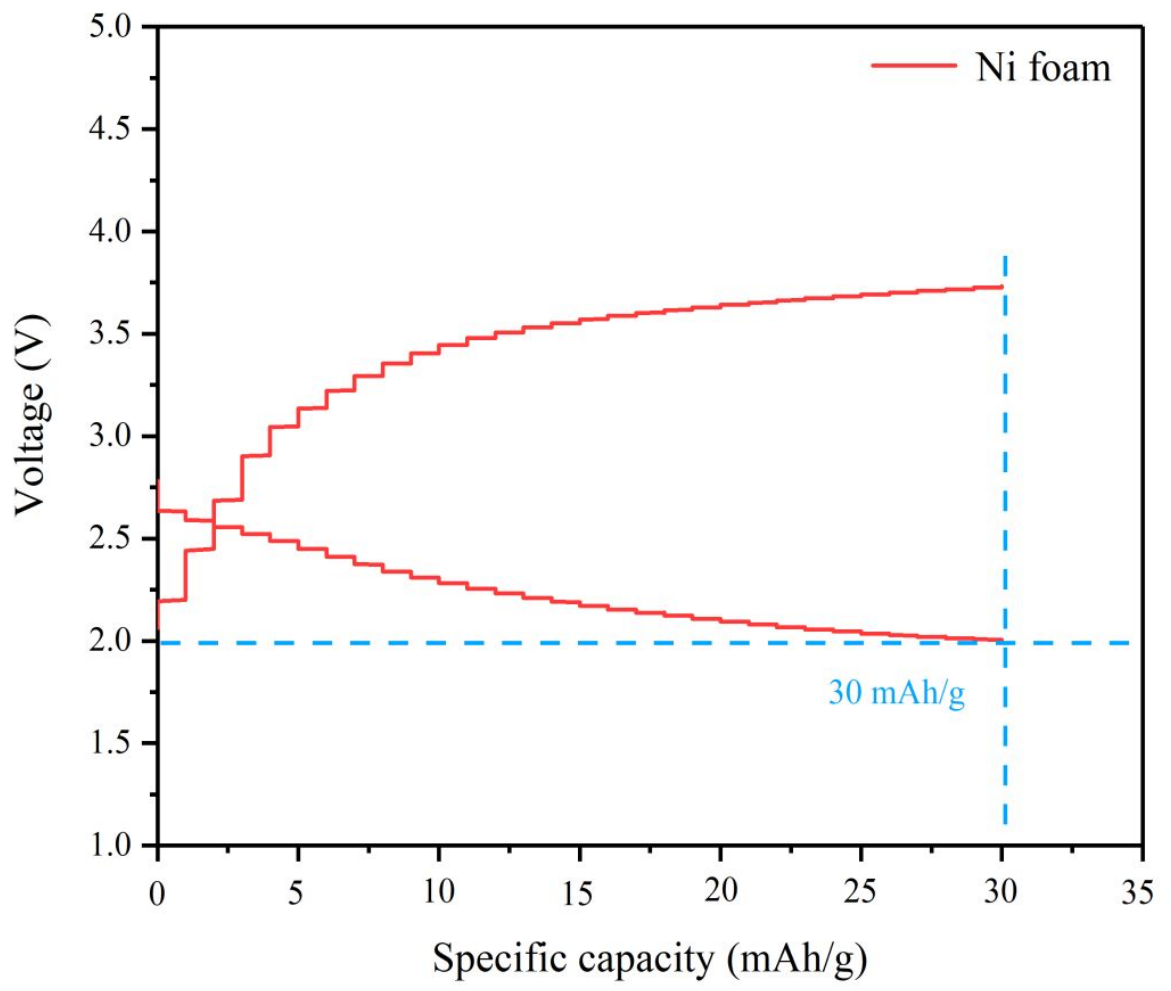

Figure S7. First charge discharge profile of $\mathrm{Ni}$ foam 


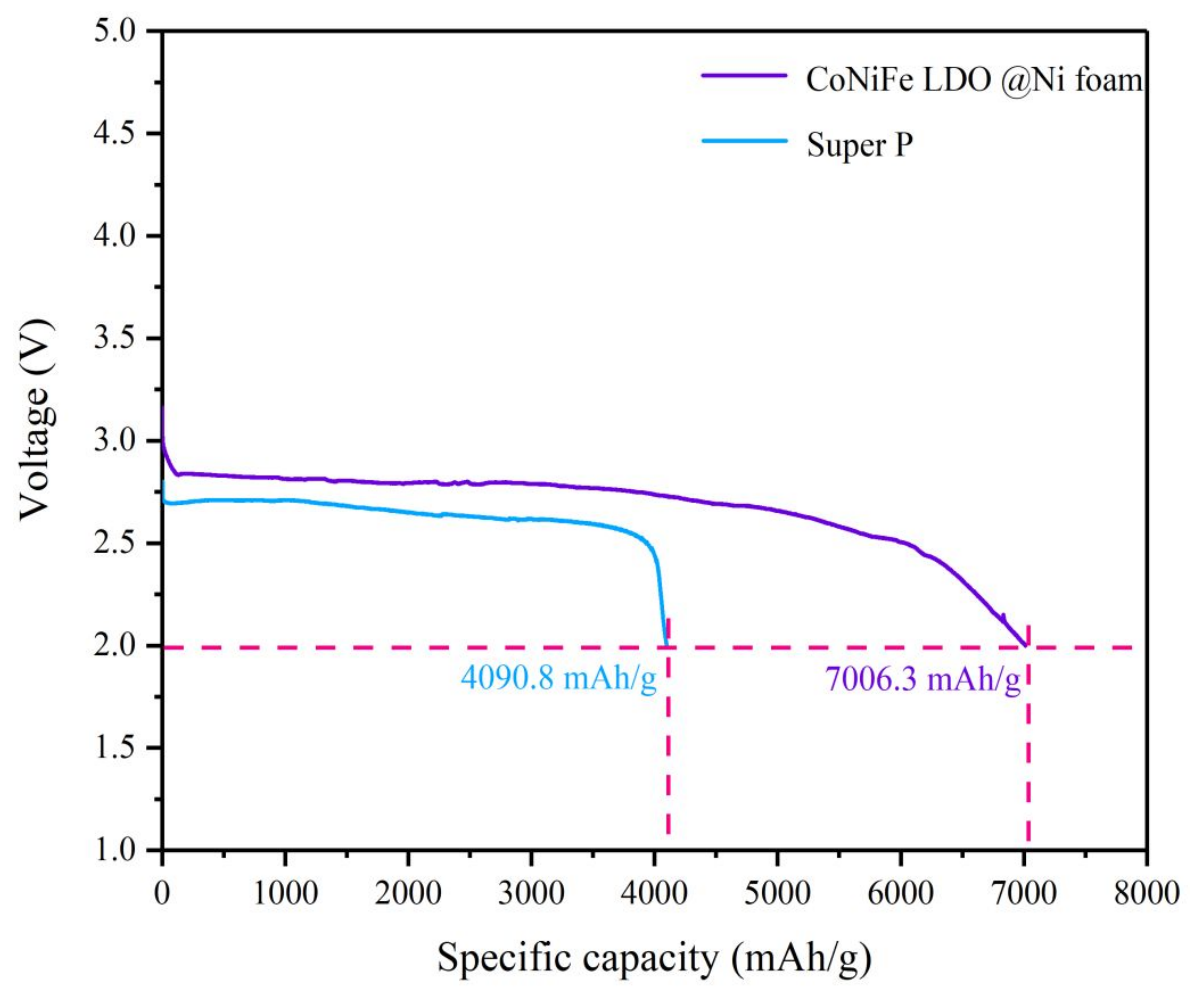

Figure S8. The full discharge profiles of CoNiFe LDO@Ni foam and super $p$ electrode. 


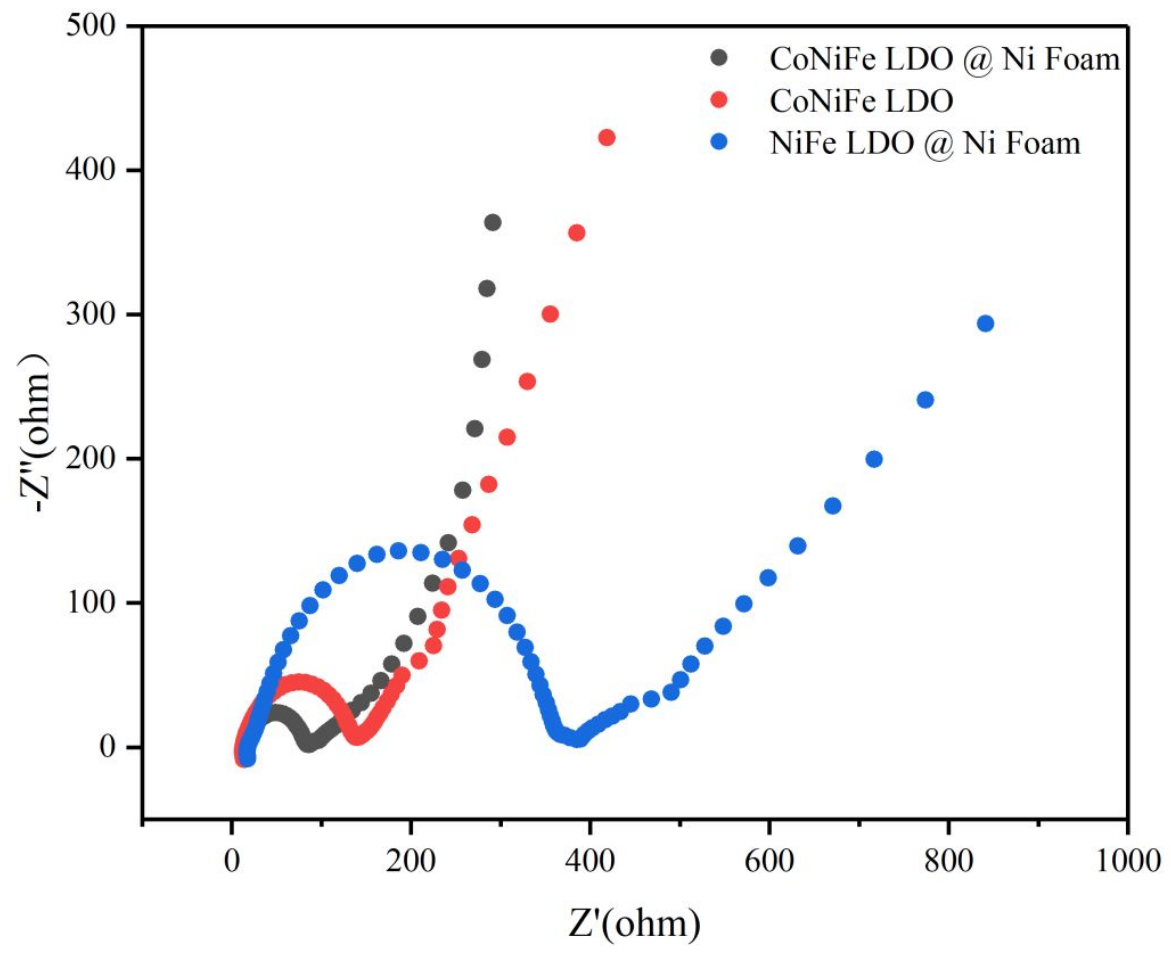

Figure S9. Electrochemical impedance spectroscopy of CoNiFe LDO@Ni foam, CoNiFe LDO, NiFe LDO@Ni Foam. 

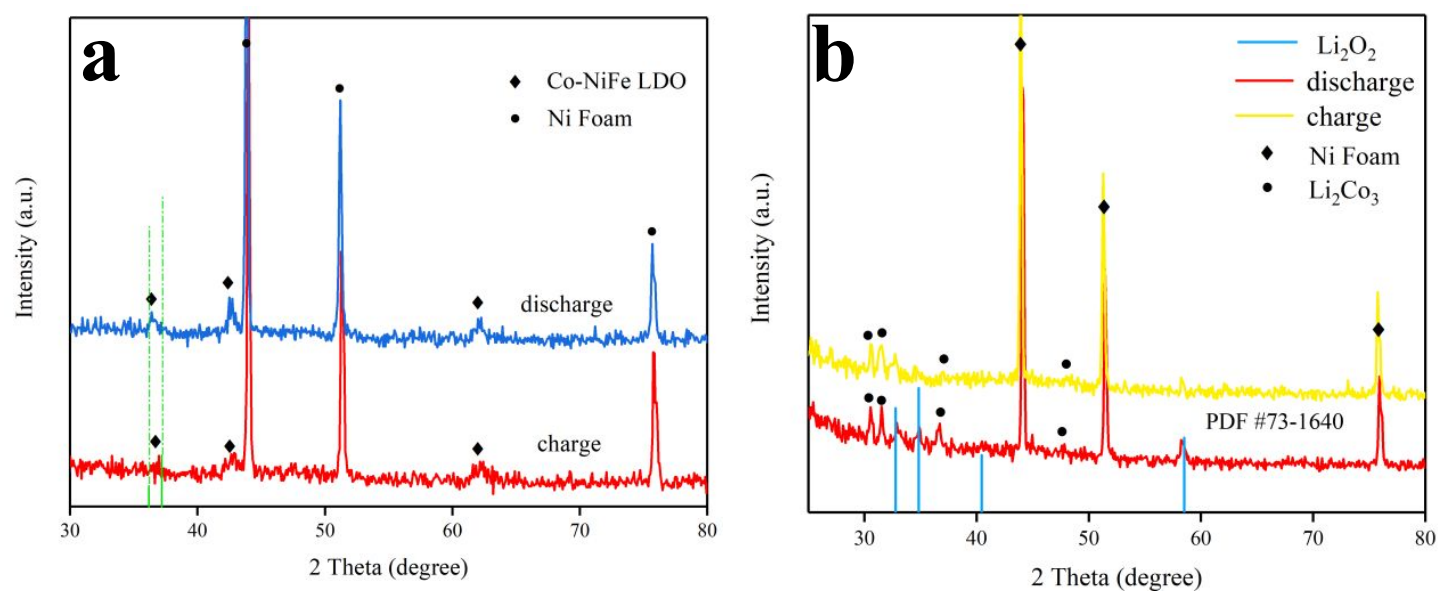

Figure S10. XRD images of CoNiFe LDO@Ni foam and super p after discharge and charge 

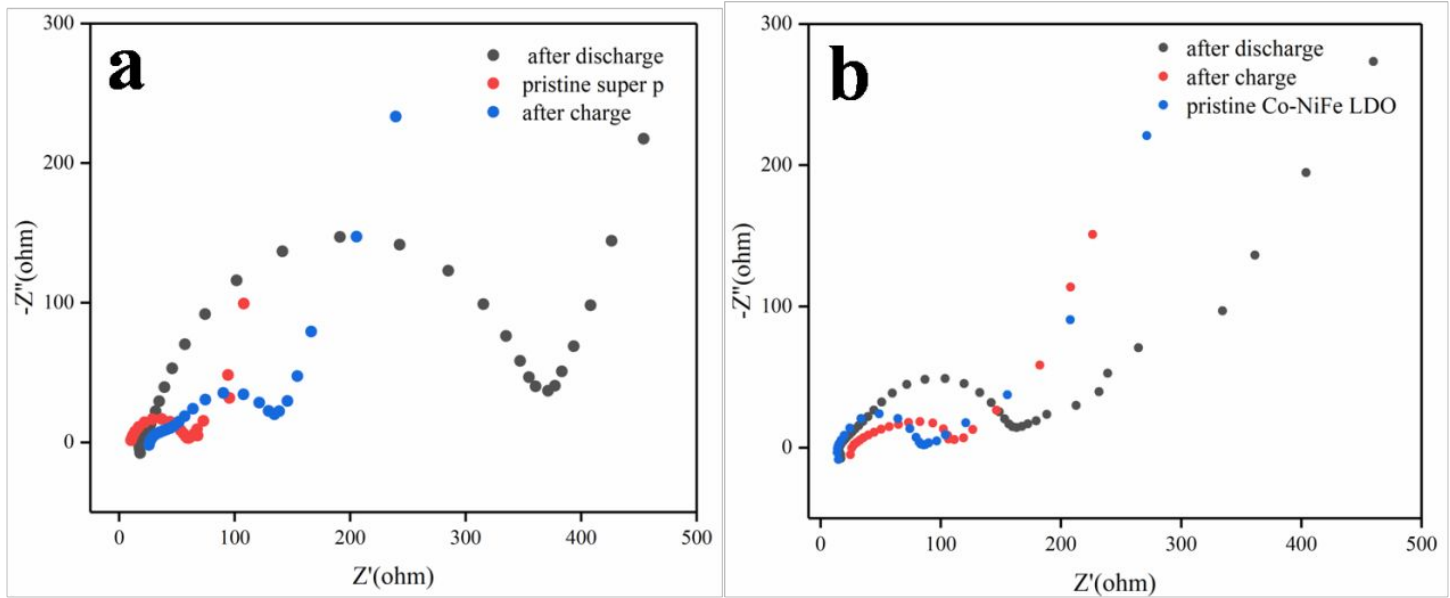

Figure S11. a) Electrochemical impedance spectroscopy of super $p$ after charge and discharge; b) Electrochemical impedance spectroscopy of Co-NiFe LDO after charge and discharge 

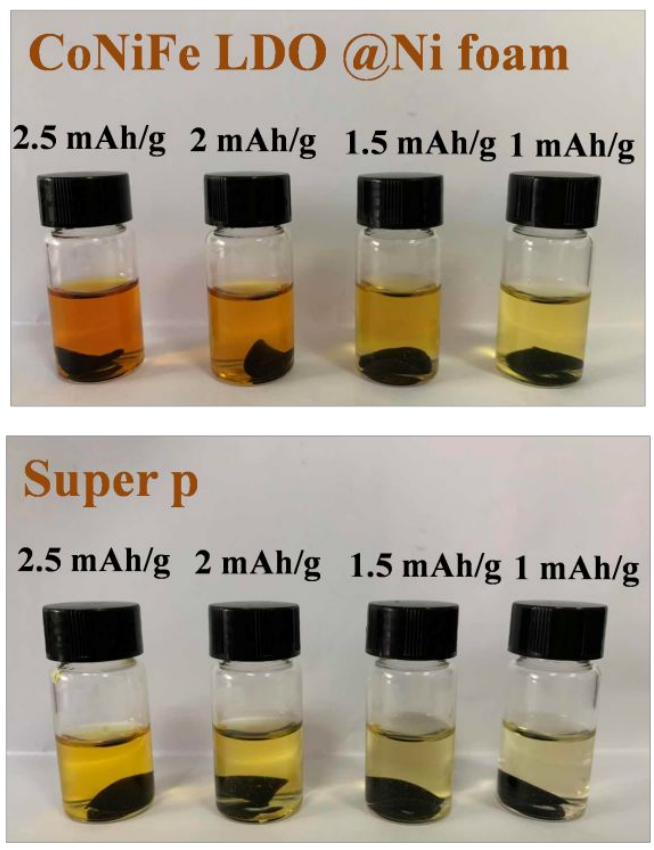

Figure S12. Pictures of CoNiFe LDO@Ni foam electrode and super p electrode immersed in $\mathrm{TiOSO}_{4}$ solution 
Table S1. The yield of $\mathrm{Li}_{2} \mathrm{O}_{2}$

Discharge capacity (mAh/g)

Yield of $\mathrm{Li}_{2} \mathrm{O}_{2}(\%)$

80.2

79.8

2

2.5

81.4

79.9 

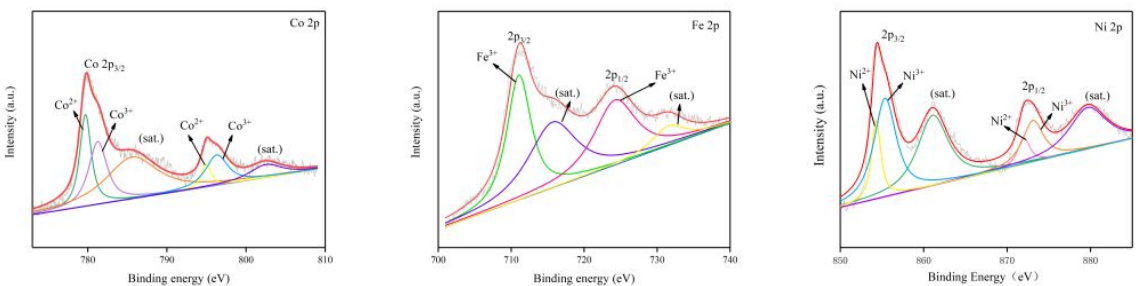

Pristine
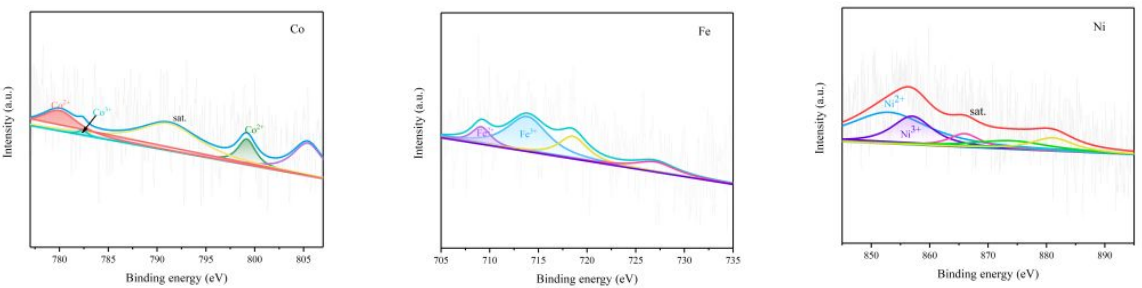

After ORR
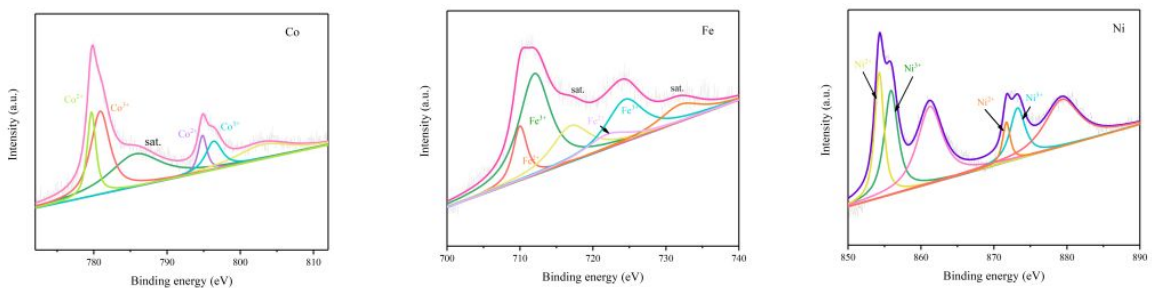

After OER

Figure S13. Valence state change of $\mathrm{Co} / \mathrm{Ni} / \mathrm{Fe}$ after charge and discharge

As displayed in Figure S13, after ORR, the signal-to-noise ratio of XPS result of each element is relatively large. The reason for this phenomenon can be attributed to the deposition of discharge products on the electrode surface during the discharge process. The XPS detection depth is generally around 2 $\mathrm{nm}$, which results in weak signal intensity of the electrode material itself while the signal of lithium oxide is stronger (Figure $7 \mathrm{c}$ in revised manuscript). However, we can still get a hint of the valence state change of $\mathrm{Ni}, \mathrm{Fe}$ and $\mathrm{Co}$ after ORR and OER. The mixed valence state of Co changed after ORR where the characteristic peak of the trivalent cobalt ion almost disappeared which proves that the trivalent cobalt ion is almost reduced to divalent cobalt ion in the ORR process. In the following OER process, the divalent cobalt ions 
are partially oxided to trivalent cobalt ions, and the ratio of the two ions $\left(\mathrm{Co}^{2+} / \mathrm{Co}^{3+}\right)$ is slightly reduced compared with the initial state, that is, the $\mathrm{Co}^{3+}$ content is relatively increased. Fe also shows significant valence change after full discharge and charge. The initial iron valence state is +3 . After discharging, the XPS signal of Fe exhibits the characteristic chemical state of divalent and trivalent coexistence. In subsequent charging process, the feature of mixed chemical state changes scarcely. As for $\mathrm{Ni}$, characteristics of the multivalent state do not change during ORR and OER, but the bivalent and trivalent ratios $\left(\mathrm{Ni}^{2+} / \mathrm{Ni}^{3+}\right)$ have obvious changed. Specifically, before discharge, the ratio of $\mathrm{Ni}^{2+}$ to $\mathrm{Ni}^{3+}\left(\mathrm{Ni}^{2+} / \mathrm{Ni}^{3+}\right)$ is less than 1 and after ORR the content of divalent nickel increases which indicates a transformation of $\mathrm{Ni3+}$ to $\mathrm{Ni}^{2+}$ takes place. In the following charge process, the $\mathrm{Ni}^{2+} / \mathrm{Ni}^{3+}$ is approximately equal to 1 , suggesting part of $\mathrm{Ni}^{2+}$ were oxidized to $\mathrm{Ni}^{3+}$. These rapid reversible chemical state changes of $\mathrm{Co} / \mathrm{Ni} / \mathrm{Fe}$ detected by XPS not only provide a rich redox couple for the reaction but also promote the interaction between electrons and donor-acceptor chemisorption sites for oxygen which benefits ORR/OER a lot. 


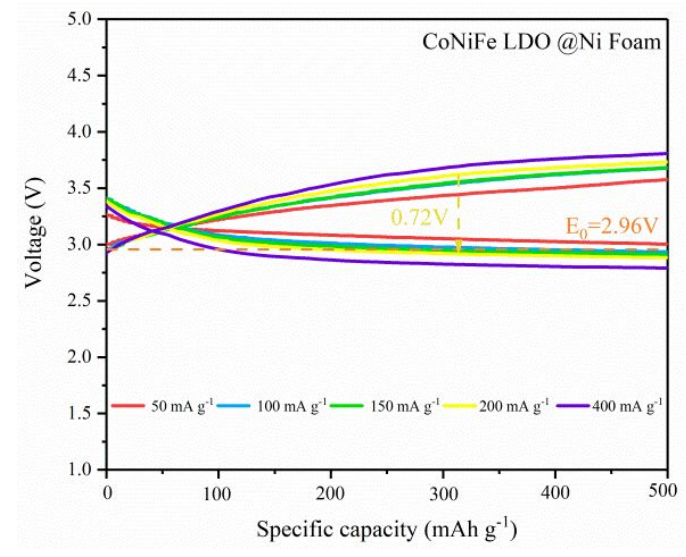

Figure S14. The overpotetial of CoNiFe LDO @Ni foam at a current density of $200 \mathrm{mAh} \mathrm{g}^{-1}$

It can be seen that the overpotential is $0.72 \mathrm{~V}$ with a current density of $200 \mathrm{~mA}$ $\mathrm{g}^{-1}$ which is close to $0.78 \mathrm{~V}\left(250 \mathrm{~mA} \mathrm{~g}^{-1}\right)$. 